\title{
Soziale Ungleichheit, psychische Gesundheit und Versorgung
}

\author{
Social Inequalites, Mental Health and Service Provision
}

Autor

Institute

\author{
Anke Bramesfeld ${ }^{1,2}$
}

Kompetenztandem Vernetzte Versorgung, Innovations-Inkubator, Leuphana-Universität Lüneburg

Institut für Epidemiologie, Sozialmedizin und Gesundheitssystemforschung, Medizinische Hochschule Hannover

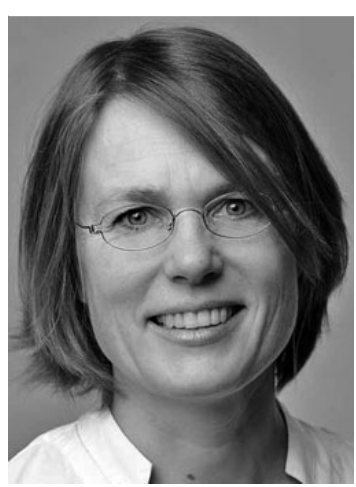

Prof. Dr. Anke Bramesfeld

\section{Bibliografie \\ Dol http://dx.doi.org/ 10.1055/s-0031-1276954 Psychiat Prax 2011; 38 : 363-365 \\ (c) Georg Thieme Verlag KG Stuttgart . New York. ISSN 0303-4259}

Korrespondenzadresse Prof. Dr. Anke Bramesfeld, MPH

Institut für Epidemiologie, Sozialmedizin und Gesundheitssystemforschung, Medizinische Hochschule Hannover, OE5410 Carl-Neuberg-Straße 1 30625 Hannover Bramesfeld.Anke@MHHannover.de
Gesundheit, einschließlich der psychischen Gesundheit ist eng verknüpft mit sozialen Parametern [1]: Das Risiko, einen vorzeitigen Tod zu sterben, ist für Menschen aus niedrigeren sozialen Schichten größer als für Menschen aus höheren Schichten. Diese Unterschiede existieren in allen Altersgruppen und für beide Geschlechter, wenn sie auch für Frauen kleiner sind und mit zunehmendem Alter abnehmen [2]. Bei den psychischen Erkrankungen finden sich Depression und Angsterkrankung in Deutschland in etwa doppelt so häufig in niedrigen sozialen Schichten wie in hohen [3].

In den letzten Jahren wird beobachtet, dass die soziale Ungleichverteilung von Gesundheit zugenommen hat [2]. Diese Beobachtung wird durch EuroStat-Daten untermauert, die eine Zunahme der Lebenserwartung in den EU-Ländern von 1997-2007 nachweisen, gleichzeitig aber auch eine Zunahme der Spannbreite der Lebenserwartung. Während die durchschnittliche Lebenserwartung eines 60 Jahre alten Mannes in den EUStaaten 1997 zwischen 15 und 20 Jahren lag, so ist im Jahr 2007 die obere Grenze auf 22 Jahre angestiegen, während sich an der unteren Grenze dieser Spannbreite fast nichts geändert hat [4]. Dies kann so interpretiert werden, dass es gesellschaftliche Gruppen gibt, die von den Prozessen, die zur Erhöhung der Lebenserwartung geführt haben, nicht erreicht werden.

Auf Bevölkerungsebene ist nur in wirklich sehr armen Ländern, wie z.B. Sierra Leone oder Senegal, der Wohlstand des Landes mit der Lebenserwartung korreliert [5]. Je höher das Bruttoinlandsprodukt und die Pro-Kopf-Ausgaben für Gesundheit sind und je näher die Lebenserwartung dem Maximum, desto uneinheitlicher ist ihre Korrelation mit der Lebenserwartung. Im Vergleich der EULänder bedeutet dies, dass Deutschland mit einem Pro-Kopf-Bruttosozialprodukt und Pro-
Kopf-Ausgaben für Gesundheit, die im Jahr 2007 denen von Schweden, Dänemark oder Frankreich vergleichbar waren, deutlich weniger gesunde Lebensjahre ab Geburt (Healthy Life Years, HLY) erreicht als diese Länder. Mit einem Bruttoinlandsprodukt von pro Kopf ca. 30000 Euro PPP $^{1}$ werden in Deutschland in etwa 56 gesunde Lebensjahre erreicht, während es in Frankreich 61 und in Dänemark und Schweden über 65 sind. Anders gesehen, Deutschland erreichte im Jahr 2007 in etwa so viele gesunde Lebensjahre wie Portugal, aber mit einem Pro-Kopf-Bruttoinlandsprodukt und Pro-Kopf-Ausgaben für Gesundheit, die um ein Drittel über denen von Portugal lagen [6].

Es gibt zunehmend Evidenz, dass es weniger der absolute Wohlstand einer Bevölkerung ist, der mit Gesundheit und Lebenserwartung assoziiert ist, als vielmehr die Verteilung der Ressourcen in einer Gesellschaft [7]. So weisen Wilkinson und Pickert nach, dass je ungleicher die Einkommen in einer Gesellschaft verteilt sind, desto geringer ist die Lebenserwartung, umso höher aber die Kindersterblichkeit, die Suizidraten, die Prävalenz an psychischen Erkrankungen einschließlich Alkohol- und Suchterkrankungen sowie die Adipositasrate. Dieser Zusammenhang ist einigermaßen linear und findet sich neben gesundheitlichen auch für weitere soziale Parameter wie die Lese- und Rechenkompetenz, Rate an Strafgefangenen, Mordrate und die Möglichkeit zur sozialen Mobilität [8]. Der Effekt, den soziale Ungleichheit auf die Chancen für Gesundheit und Wohlergehen hat, trifft alle sozialen Schichten: So hat ein Mensch aus einer hohen sozialen Schicht in einem Land mit größerer sozialer Ungleichheit immer noch eine geringere Chance für Gesundheit und eine geringere Lebenserwartung als ein

\footnotetext{
${ }^{1}$ PPP: Pruchaising Power Parity, unter Berücksichtigung der Kaufkraft eines Landes angeglichener Euro-Wert.
} 


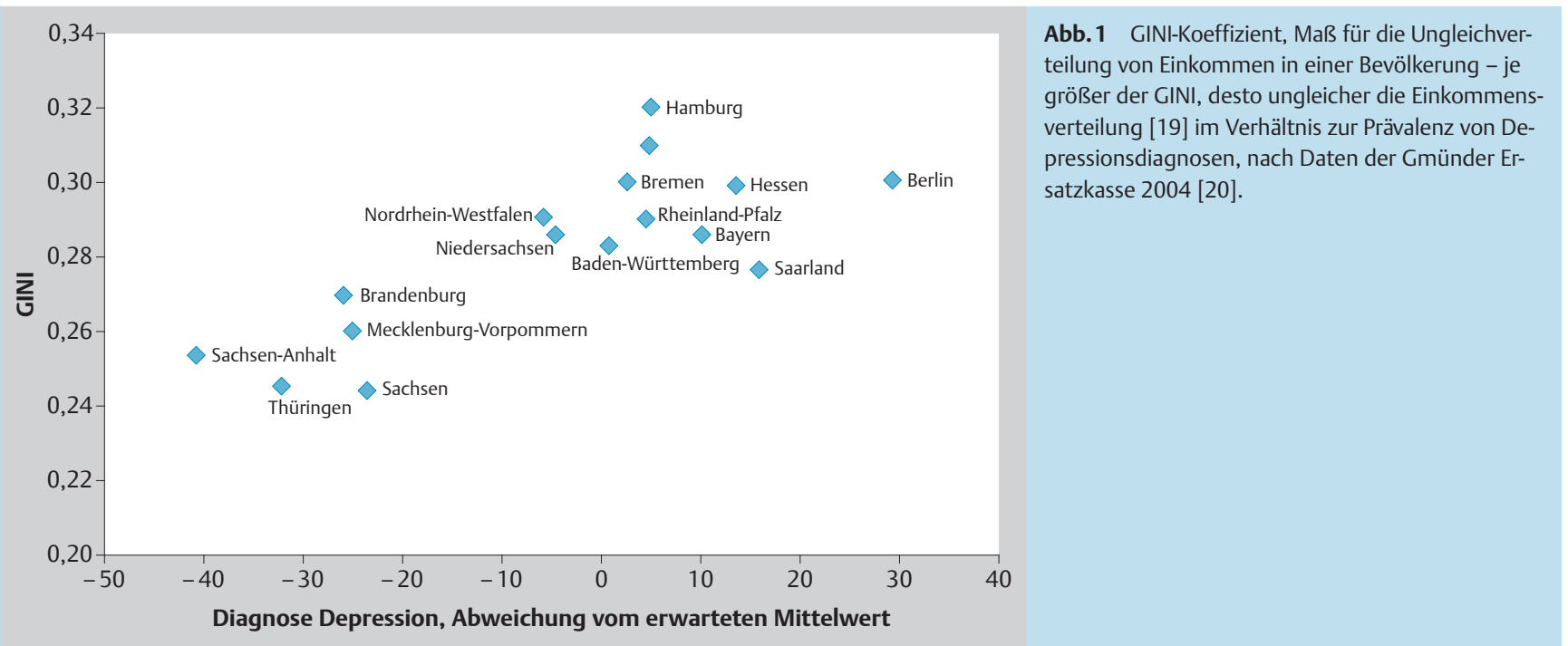

Mensch aus einer vergleichbaren sozialen Schicht in einem sozial gleicheren Land [9].

Der von Wilkinson und Pickert dargestellte Zusammenhang zwischen sozialer Ungleichheit und der Prävalenz psychischer Erkrankungen lässt sich auch mit eigenen Daten für Deutschland nachvollziehen. Es zeigt sich, dass die Prävalenz an Depressionsdiagnosen (als angenommenes Proxy für die Prävalenz von Depressionserkrankung) assoziiert ist mit der Ungleichheit der Verteilung der Einkommen in den Bundesländern ( $\bullet$ Abb. 1).

Welches aber sind die Faktoren, die zwischen der Ungleichverteilung der Einkommen in einer Bevölkerung und Krankheit, insbesondere psychischer Morbidität vermitteln? Drei Hypothesen werden diskutiert: 1 . die Sozialkapital-Hypothese, 2. die Statusangst-Hypothese und 3. die neomaterialistische Hypothese.

Die Sozialkapital-Hypothese geht davon aus, dass Gesellschaften mit einem besseren Vertrauen und Zusammenhalt ihrer Mitglieder mehr Sorge um das gesundheitsrelevante Verhalten ihrer Mitglieder tragen. Dies erleichtert den Zugang zu Versorgung für alle und führt zu besseren öffentlichen Versorgungsdiensten, was wiederum allen Mitgliedern der Gesellschaft nutzt [10].

Die Statusangst-Hypothese geht davon aus, dass in ungleicheren Gesellschaften mehr Wettbewerb um den Status und eine größere Ausdifferenzierung der Schichten existiert. Diejenigen, die am gesellschaftlichen unteren Rand leben, sind durch die Selbstwahrnehmung ihres schlechten Status größerem Stress ausgesetzt, was die Gesundheit beeinträchtigt [11]. Darüber hinaus geht die Statusangst-Hypothese davon aus, dass Status-Konkurrenz, den sozialen Zusammenhalt und das Vertrauen in einer Gesellschaft beeinträchtigen. Hier überschneidet sich die Statusangst-Hypothese mit der Sozialkapital-Hypothese.

Die neomaterialistische Hypothese schließlich geht davon aus, dass die Ungleichverteilung in einer Gesellschaft das Resultat von historischen, kulturellen und politisch-ökonomischen Prozessen ist. Diese Prozesse beeinflussen nicht nur das private Einkommen von Individuen, sondern auch die öffentliche Infrastruktur in Bezug auf Zugang und Qualität von Bildung, Gesund- heitsversorgung, Transport, Wohnraum, Arbeitsschutz und anderem [12].

Welche der 3 Hypothesen am besten geeignet ist, den Zusammenhang zwischen sozialer Ungleichheit und psychischer Gesundheit zu erklären, bleibt kontrovers. Untersuchungen für Europa mit den Daten des European Quality of Life Surveys (EQLS) [13] erbrachten widersprüchliche Ergebnisse [14,15].

Was bedeutet die Berücksichtigung der sozialen Ungleichheit für die Gesundheits- und insbesondere für die psychiatrische Versorgung?

Die Kompetenz soziale Ungleichheit als Basis für ungleiche Gesundheitschancen in einer Gesellschaft anzugehen, und die Verteilung von Gütern und Wohlstand zu regeln liegt außerhalb des Wirkungseinflusses des Gesundheitswesens. Der Zugang zu Versorgung, und die Qualität der sozialen Infrastruktur fällt jedoch in die Zuständigkeit des Gesundheitssystems und damit auch der psychiatrischen Versorgung. Guter Zugang zu (psychiatrischer) Versorgung gerade für die meist schwer erreichbaren Gruppen [16] aus niedrigeren sozialen Schichten und Vernetzung mit anderen Versorgungssektoren über den medizinischen Sektor hinaus, im Sinne von Schaffen eines „Sozialkapitals in der Versorgung“ $[17,18]$, sollte mehr als bisher als relevanter Parameter für die Qualität von psychiatrischer Versorgung etabliert werden.

\section{Literatur}

1 Wilkinson R, Marmot M. The solid facts. Social determinants of health. 2 ed. Kopenhagen: World Health Organisation; 2003

2 Mackenbach J. Health Inequalities: Europe in Profile. 2006; http://ec. europa.eu/health/ph_determinants/socio_economics/documents/ ev_060302_rd06_en.pdf

3 Fryers T, Melzer D, Jenkins $R$ et al. The distribution of common mental disorders: social inequalities in Europe. Clin Pract Epidemol Ment Health 2005; $1: 14$

4 Paoli F. Increase in life expectancy at age 60. EuroStat Data; 2011 1-12010

5 UC Atlas on inequality. Life expectancy vs. spending. http://ucatlas. ucsc.edu/spend.php 13-9-2006

6 OECD. Health at a Glance: Europe 2010. Berlin: OECD Publishing; 2010

7 Subramanian SV, Kawachi I. Income inequality and health: what have we learned so far? Epidemiol Rev 2004; 26: 78-91 
8 Wilkinson RG, Pickett $K$. The problems of relative deprivation: Why some societies do better than others. Soc Sci Med 2007; 65: 19651978

9 Wilkinson RG, Pickett $K$. The Spirit Level: Why Equality is Better for Everyone. London: Pinguin Books; 2010

10 Kawachi I. Social capital and community effects on population and individual health. Ann N Y Acad Sci 1999; 896: 120-130

11 Wilkinson RG, Pickett KE. Income inequality and population health: a review and explanation of the evidence. Soc Sci Med 2006; 62: 17681784

12 Lynch JW, Smith GD, Kaplan GA et al. Income inequality and mortality: importance to health of individual income, psychosocial environment, or material conditions. BMJ 2000; 320: 1200-1204

13 Anderson R, Mikulic B, Vermeylen G et al. Second European Quality of Life Survey. Overview. European Foundation for the Improvement of Living and Working Conditions. 2009; http://www.eurofound.europa. eu/pubdocs/2009/02/en/2/EF0902EN.pdf

14 Layte R, Maitre B. The Association Between Income Inequality and Mental Health: Social Cohesion or Social Infrastructure? ESRI Working Paper 328. Pub Date 5-11-2009. ESRI. http://www.esri.ie/UserFiles/ publications/20091105141934/WP328.pdf
15 Layte $R$. The Association between Income Inequality and Mental Health: Testing Status Anxiety, Social Capital and Neo-Materialist Explanations. Europ Soc Rev 2011; e-first: DOI: 10.1093/esr/jcr012

16 Schouler-Ocak M, Bretz HJ, Hauth I et al. Patienten mit Migrationshintergrund in Psychiatrischen Institutsambulanzen - ein Vergleich zwischen Patienten mit türkischer und osteuropäischer Herkunft sowie Patienten ohne Migrationshintergrund. Psychiat Prax 2010; 37: 384390

17 Rosenheck R, Morrissey J, Lam J et al. Service delivery and community: social capital, service systems integration, and outcomes among homeless persons with severe mental illness. Health Serv Res 2001; 36: 691-710

18 Battams S, Baum F. What policies and policy processes are needed to ensure that people with psychiatric disabilities have access to appropriate housing? Soc Sci Medi 2010; 70: 1026-1034

19 Statistische Ämter des Bundes und der Länder. Gini-Koeffizient der Äquivalenzeinkommen. Sozialberichterstattung 20-8-2010

20 Bramesfeld A, Grobe T, Schwartz F. Prevalence of depression diagnosis and prescription of antidepressants in East and West Germany: an analysis of health insurance data. Soc Psychiatry Psychiatr Epidemiol 2010; 45: 329-335 